\title{
Associação entre acrocórdons e resistência à insulina
}

\author{
Association between skin tags and insulin resistance
}

\author{
Andréia de Almeida Tamega ${ }^{1}$ \\ Marcelo Massaki Guiotoku ${ }^{3}$ \\ Hélio Amante Miot ${ }^{5}$
}

\author{
Adriana Milanezi Pinheiro Aranha ${ }^{2}$ \\ Luciane Donida Bartoli Miot ${ }^{4}$
}

Resumo: FundAmENTOS: Acrocórdons são lesões dermatológicas comuns na população e estão associados ao diabetes mellitus, à obesidade, à resistência insulínica e à aterosclerose. A identificação precoce de pacientes com resistência insulínica pode ter papel preventivo primário.

Овјетіvо: Avaliar a associação entre presença de acrocórdons cervicais ou axilares e resistência insulínica.

MÉTODOs: Estudo transversal com pacientes dermatológicos adultos atendidos em hospital universitário. Casos foram definidos como portadores de mais de cinco acrocórdons cervicais e/ou axilares. A resistência insulínica foi estimada pelo índice HOMA-IR. Resultados foram ajustados pelas demais covariáveis de risco para resistência insulínica conhecidos, a partir de regressão logística múltipla.

RESULTADOS: Avaliaram-se 98 casos e 103 controles, que não diferiram entre si quanto à idade ou ao gênero. Acrocórdons se associaram diretamente aos valores de HOMA-IR (Odds Ratio $=1,4$ ), hipertrigliceridemia e índice de massa corpórea, independentemente do ajuste por diabetes mellitus, idade, fototipo, gênero, história de diabetes mellitus familiar e relação cintura/quadril. Níveis qualitativamente elevados de HOMA-IR $(>3,8)$ também evidenciaram associação significativa (Índice de probabilidade $=7,5$ ).

CONCLuSÕES: Presença de múltiplos acrocórdons se associou à resistência insulínica, independentemente dos demais fatores de risco.

Palavras-chave: Diabetes Mellitus; Estudos transversais; Obesidade; Resistência à insulina

\begin{abstract}
Background: Skin tags are dermatological lesions commonly found in the general population and have been associated with diabetes mellitus, obesity, insulin resistance and atherosclerosis. Early detection of patients with insulin resistance may play an important preventive role.

Objective: To evaluate the association between skin tags in the neck or axillary regions and insulin resistance.

Methods: A cross-sectional study involving adult patients receiving care at a university teaching hospital. Cases were defined as patients with $>5$ skin tags in the neck region and/or axillae. Insulin resistance was estimated using the HOMA-IR index. Results were adjusted for the other known covariates of risk for insulin resistance using a multiple logistic regression model.

Results: Ninety-eight cases and 103 controls were evaluated. There was no difference between the groups with respect to age or gender. Skin tags were directly associated with HOMA-IR values (odds ratio $=1.4$ ), hypertriglyceridemia and body mass index, irrespective of adjustment for diabetes mellitus, age, skin phototype, gender, family history of diabetes mellitus or hip/waist ratio. Qualitatively elevated HOMA-IR levels $(>3.8)$ were also significantly associated (odds ratio $=7.5$ ).

Conclusions: The presence of multiple skin tags was strongly associated with insulin resistance irrespective of other risk factors.

Keywords: Cross-sectional studies; Diabetes Mellitus; Insulin resistance; Obesity;
\end{abstract}

Recebido em 30.07.2009.

Aprovado pelo Conselho Consultivo e aceito para publicação em 18.12.2009.

* Trabalho realizado no Departamento de Dermatologia e Radioterapia da Faculdade de Medicina de Botucatu da Universidade Estadual Paulista (FMB-Unesp)

- Botucatu (SP), Brasil.

Conflito de interesse: Nenhum / Conflict of interest: None

Suporte financeiro: Nenhum / Financial funding: None

Dermatologista; mestranda em Patologia da Faculdade de Medicina de Botucatu da Universidade Estadual Paulista (FMB-Unesp) - Botucatu (SP), Brasil.

Nutricionista colaboradora do Departamento de Dermatologia e Radioterapia da Faculdade de Medicina de Botucatu da Universidade Estadual Paulista (FMBUnesp) - Botucatu (SP), Brasil.

Residente do Departamento de Dermatologia e Radioterapia da Faculdade de Medicina de Botucatu da Universidade Estadual Paulista (FMB-Unesp) - Botucatu (SP), Brasil.

Dermatologista do Departamento de Dermatologia e Radioterapia da Faculdade de Medicina de Botucatu da Universidade Estadual Paulista (FMB-Unesp) Botucatu (SP), Brasil.

Professor assistente doutor do Departamento de Dermatologia e Radioterapia da Faculdade de Medicina de Botucatu da Universidade Estadual Paulista (FMBUnesp) - Botucatu (SP), Brasil. 


\section{INTRODUÇÃO}

Acrocórdons (skin tags) representam os tumores cutâneos fibroepiteliais mais comuns; são pólipos benignos adquiridos que surgem nas dobras naturais da pele, como as regiões cervical, axilar, inguinal, crural, perineal, inframamária, as pálpebras e o sulco interglúteo. ${ }^{1,2}$ São pápulas pedunculadas e amolecidas que se protruem a partir da superfície da pele (Figura 1). Únicas ou múltiplas, elas variam entre $2 \mathrm{~mm}$ e $10 \mathrm{~mm}$, com tendência a crescimento progressivo, sem involução espontânea. São normocrômicas ou hipercrômicas e, apesar de assintomáticas, são queixas frequentes no consultório dermatológico por razões estéticas ou, ainda, por traumatismo nas roupas ou jóias. ${ }^{1.3} \mathrm{Em}$ 2006, de acordo com a região do País, estimou-se que de $0,9 \%$ a $1,2 \%$ dos diagnósticos em consultas dermatológicas no Brasil se referiam a acrocórdons. ${ }^{4}$ Estes são extremamente comuns na população adulta maior que 40 anos (46\%), aumentando sua incidência entre os idosos (59\% aos 70 anos). ${ }^{3}$ Apresentam componente familiar, mas o padrão genético de segregação não foi ainda definido, assim como os aspectos étnicos, não havendo predileção por gênero. Associa-se à gravidez, à acromegalia, aos pólipos intestinais (sintomáticos), à dislipidemia, à obesidade, ao diabetes mellitus (DM), à aterosclerose e a várias síndromes: ovários policísticos, Birt-Hogg-Dubé e Cowden. O ácido desoxirribonucléico (DNA) do buman papillomavirus (HPV) foi evidenciado em $88 \%$ das lesões de acrocórdons. ${ }^{5.9}$ Variações hormonais estrogênicas e hormônios tróficos, como IGF-1 (insulin-like growth factor-1), insulina, TGF $\alpha$ (transforming growth factor- $\alpha$ ) e fator de crescimento epidérmico (EGF), estão envolvidos na gênese e no desenvolvimento dos acrocórdons. ${ }^{8-10}$

A resistência periférica à insulina (RI) é alteração metabólica sistêmica que tem importante impacto na mortalidade geral da população, favorecendo eventos

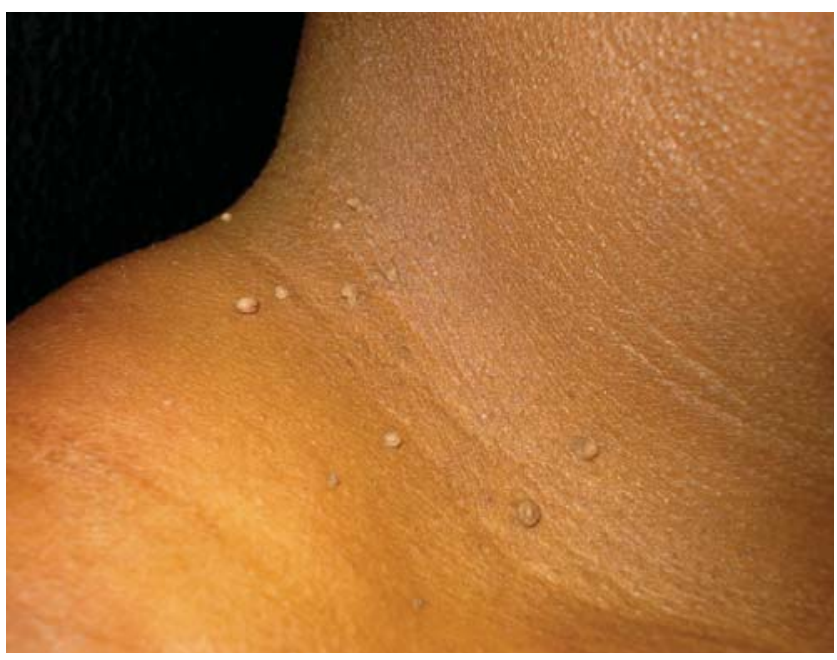

FigurA 1: Acrocórdons cervicais múltiplos cardiovasculares e certas neoplasias malignas. A RI constitui o elemento central da síndrome metabólica (síndrome $\mathrm{X}$ ), que afeta $20 \%$ a $30 \%$ das pessoas em países industrializados e se associa a fatores de risco para a aterosclerose, como o DM tipo II, a hipertensão arterial sistêmica, a obesidade (abdominal) e a dislipidemia. Ademais, a RI representa, com o tabagismo, o alcoolismo e a violência no trânsito, um dos fatores modificáveis mais importantes para a promoção da saúde e a redução geral da mortalidade populacional. ${ }^{11-13}$

Tendo-se em vista que a RI se desenvolve antes do aparecimento e da possibilidade de detecção das doenças associadas, a identificação e o tratamento precoces desses pacientes podem exercer importante papel preventivo primário, incentivando a mudança do estilo de vida e o tratamento específico.

Há diversas manifestações cutâneas que se associam à RI, como pseudoacantose nigricans, hirsutismo, acne, hidradenite supurativa, oleosidade, alopecia, papilose digital e acrocórdons. ${ }^{13-15}$

Pode-se estimar a RI a partir do teste oral de tolerância à glicose, da curva glicêmica, dos índices que comparam a produção de insulina basal e a glicemia de jejum, como os índices HOMA-IR (bomeostatic model assessment) e Quick, além dos elementos fenotípicos, como o índice de massa corporal (IMC) e a medida da relação cintura/quadril. O índice HOMA-IR é largamente utilizado em testes clínicos e se baseia no produto da glicemia de jejum e insulina basal. ${ }^{16,17}$

Ainda não foi estudada a associação entre múltiplos acrocórdons e RI na população brasileira, e esse é o objetivo deste estudo.

\section{CASUÍSTICA}

Estudo transversal com 201 pacientes adultos, esclarecidos e concordantes, do ambulatório de dermatologia do Hospital das Clínicas da Faculdade de Medicina de Botucatu (FMB-Unesp), no período de fevereiro de 2008 a fevereiro de 2009.

Definiram-se os casos como os portadores de mais de cinco acrocórdons cervicais ou axilares; os controles abrangeram os demais pacientes dermatológicos sem nenhuma lesão no momento do exame e sem história de exérese prévia de acrocórdons. Excluíram-se pacientes que referiam: acromegalia, feocromocitoma, hemocromatose, síndrome de Cushing e glucagonoma.

Todos os pacientes foram entrevistados e examinados individualmente, e o mesmo investigador mediu suas variáveis antropométricas. Os exames laboratoriais (glicemia de jejum e insulina basal) foram colhidos no mesmo laboratório. A principal variável independente foi o índice HOMA-IR e a variável dependente da pesquisa foi a presença de acrocórdons cervicais e axilares. 
A associação entre os grupos foi avaliada, inicialmente, por modelo bivariado e depois ajustada pelas covariáveis: idade, gênero, fototipo, relação cintura/quadril, índice de massa corpórea, presença de pseudoacantose nigricans, DM, DM familiar, hipercolesterolemia, hipertrigliceridemia, glicemia, insulina basal e HOMA-IR, a partir de um modelo de regressão logística múltipla condicional. O DM, a hipercolesterolemia e a hipertrigliceridemia foram definidos nos pacientes que referiram tais diagnósticos ou que apresentaram exames em jejum prévios ou atuais, no laboratório da instituição, com níveis maiores que: glicemia de 126 (mg/dl), colesterol total e triglicerídeos de 200 (mg/dl), valores considerados normais para as referências do laboratório utilizado.

Compararam-se as frequências das variáveis categóricas pelos testes do qui-quadrado (de tendência, se indicado) e Fisher. Variáveis contínuas foram descritas como médias ou medianas e comparadas pelos testes t de Student, Mann-Whitney e KruskalWallis. Estimou-se a normalidade das distribuições pelo teste de Shapiro-Wilk e construiu-se o modelo logístico multivariado a partir de algoritmo de inclusão sequencial (stepwise forward) das covariáveis que influenciaram a verossimilhança, com significância inferior a 0,3 em cada passo. Estimou-se a associação entre as variáveis a partir do Odds Ratio (OR) e de seu intervalo de confiança de 95\% (IC 95\%).

A amostragem foi determinada a partir de um pré-teste com 50 casos e 50 controles, satisfazendo um poder de 0,9 e nível alfa de 0,01 para a comparação bivariada das proporções dos níveis de HOMA-IR alterados, e foi ampliada para incluir mais de cinco casos para cada covariável de ajuste no modelo logístico final.

Realizou-se a tabulação e a análise dos dados pelos software MS Excel 2003 e SPSS $17 .{ }^{18}$ Considerouse significativo o valor de $\mathrm{p}<0,05$ bicaudal.

\section{RESULTADOS}

Entrevistaram-se 98 casos e 103 controles. Nenhum paciente se recusou a participar do estudo, entretanto, somente 133 deles $(66,2 \%)$ realizaram a coleta de exames laboratoriais. Os pacientes que não coletaram os exames laboratoriais não diferiram dos que coletaram quanto ao grupo (casos e controles), gênero, idade e IMC, o que não impactou a validade interna do estudo. Além disso, a amostra de 61 casos e 72 controles que coletaram os exames contemplou as necessidades estabelecidas previamente. A tabela 1 demonstra as frequências das variáveis e a análise bivariada entre casos e controles.

Acrocórdons cervicais ocorreram em 85,7\% dos casos, axilares, em 62,2\% e, em 28,6\%, outras topografias. Houve concomitância entre lesões cervicais e axilares em 53,1\% dos casos.
O gênero feminino prevaleceu em ambos os grupos e as idades foram características de pacientes adultos entre 30 e 70 anos. Identificou-se prevalência de fototipo mais claros entre os controles, indicando a necessidade de ajuste posterior na análise multivariada.

O grupo portador de acrocórdons se associou primariamente e de forma direta ao IMC, à relação cintura/quadril, ao DM, à hipertrigliceridemia, à presença de pseudoacantose nigricans, aos níveis de glicemia, insulina e HOMA-IR (quantitativo e qualitativo). A forma mais identificada de pseudoacantose nigricans, em ambos os grupos, foi a axilar; entretanto, o acometimento cervical foi o mais associado à presença de acrocórdons.

A análise multivariada corroborou o achado de maiores níveis de HOMA-IR entre os casos, demonstrando associação independente do ajuste das covariáveis (Tabela 2). Manteve-se significativa a associação entre acrocórdons e IMC, hipertrigliceridemia e história de DM familiar.

Quando considerado apenas o valor qualitativo de HOMA-IR (> 3,8), observou-se elevação em 31,1\% dos casos e $2,8 \%$ dos controles, o que reiterou a associação positiva das variáveis. $\mathrm{O} O R$ ajustado por idade, fototipo, gênero, DM, IMC, relação cintura/quadril, DM familiar e hipertrigliceridemia foi de 7,5 (IC 95\% 1,38 a 40,9; p < 0,02).

A análise comparativa dos pacientes com acrocórdons cervicais, axilares ou de ambos os locais não revelou diferenças quanto à estimativa do HOMA-IR $(\mathrm{p}>0,3)$.

\section{DISCUSSÃO}

Identificou-se associação independente entre a presença de mais de cinco acrocórdons e a elevação de 1,4 unidades do índice HOMA-IR em pacientes dermatológicos. A significância da associação com IMC e hipertrigliceridemia encontrada neste estudo fortalece o conceito de que acrocórdons podem representar um marcador de resistência insulínica.

Gênero masculino e idade são outros fatores de risco conhecidos que não se confirmaram nessa amostra, assim como foi contraditória a maior frequência de história familiar de DM entre os controles. Tais elementos precisam ser investigados em outros estudos, bem como a possibilidade de viés de memória.

A síndrome metabólica é uma alteração sistêmica que promove diversas alterações tróficas no organismo. Em estudo de base hospitalar envolvendo 118 pacientes com acrocórdons, identificou-se RI ou DM em 40,6\% deles. ${ }^{19}$ Neste trabalho, como se avaliou uma população ambulatorial normal, a prevalência geral de níveis elevados de HOMA-IR foi baixa, porém, com tendência a valores mais elevados entre os casos. 
TABela 1: Principais características demográficas, antropométricas e laboratoriais dos grupos estudados

\begin{tabular}{|c|c|c|c|c|c|c|c|c|}
\hline & & Casos & $\%$ & Controles & $\%$ & OR & IC $95 \%$ & $\mathbf{p}$ \\
\hline N. & & 98 & & 103 & & & & \\
\hline \multirow[t]{2}{*}{ Gênero } & Feminino & 73 & 74,5 & 83 & 80,6 & 0,70 & 0,36 a 1,37 & $0,30 *$ \\
\hline & Masculino & 25 & 25,5 & 20 & 19,4 & & & \\
\hline \multirow[t]{2}{*}{ Idade (anos) } & Média & 51,3 & & 52,1 & & - & - & $0,67^{\# \#}$ \\
\hline & $\mathrm{dp}$ & 11,2 & & 15,2 & & & & \\
\hline \multirow[t]{2}{*}{ IMC $\left(\mathbf{k g} / \mathbf{m}^{2}\right)$} & Mediana & 31,1 & & 26,1 & & - & - & $<0,01^{s}$ \\
\hline & $\begin{array}{l}\text { Desvio } \\
\text { interquartílico }\end{array}$ & 8,1 & & 5,6 & & & & \\
\hline \multirow{6}{*}{ fototipo } & I & 7 & 7,1 & 19 & 18,4 & - & - & $0,04 * *$ \\
\hline & II & 26 & 26,5 & 31 & 30,1 & & & \\
\hline & III & 38 & 38,8 & 29 & 28,2 & & & \\
\hline & IV & 13 & 13,3 & 12 & 11,7 & & & \\
\hline & $\mathrm{V}$ & 9 & 9,2 & 11 & 10,7 & & & \\
\hline & VI & 5 & 5,1 & 1 & 1,0 & & & \\
\hline Relação & Média & 0,92 & & 0,88 & & - & - & $<0,01^{\# \#}$ \\
\hline Cintura/Quadril & Desvio padrão & 0,08 & & 0,08 & & & & \\
\hline \multirow[t]{2}{*}{ Diabetes mellitus } & Sim & 22 & 22,4 & 9 & 8,7 & 3,02 & 1,31 a 6,95 & $0,01^{*}$ \\
\hline & Não & 76 & 77,6 & 94 & 91,3 & & & \\
\hline \multirow{2}{*}{$\begin{array}{l}\text { Diabetes mellitus } \\
\text { Familiar }\end{array}$} & Sim & 45 & 45,9 & 60 & 58,3 & 0,61 & 0,36 a 1,11 & $0,08 *$ \\
\hline & & Não & 53 & 54,1 & 43 & 41,7 & & \\
\hline \multirow{5}{*}{$\begin{array}{l}\text { Pseudoacantose } \\
\text { nigricans }\end{array}$} & Ambos & 15 & 15,3 & 3 & 2,9 & 6,02 & 1,69 a 21,52 & $<0,01^{\#}$ \\
\hline & Axilar & 48 & 49,0 & 26 & 25,2 & 2,84 & 1,50 a 4,95 & $<0,01^{*}$ \\
\hline & Cervical & 16 & 16,3 & 3 & 2,9 & 6,50 & 1,83 a 23,10 & $<0,01^{\#}$ \\
\hline & Axilar OU & 48 & 49,0 & 26 & 25,2 & 2,84 & 1,57 a 5,16 & $<0,01 *$ \\
\hline & Cervical & & & & & & & \\
\hline \multirow{2}{*}{$\begin{array}{l}\text { Hipertrigliceridemia } \\
(>200 \mathrm{mg} / \mathrm{dl})\end{array}$} & Sim & 30 & 30,6 & 15 & 14,6 & 2,59 & 1,31 a 5,12 & $0,01 *$ \\
\hline & Não & 68 & 69,4 & 88 & 85,4 & & & \\
\hline \multirow{2}{*}{$\begin{array}{l}\text { Hipercolesterolemia } \\
\text { (> 200mg/dl) }\end{array}$} & Sim & 46 & 46,9 & 40 & 38,8 & 1,39 & 0,80 a 2,44 & $0,25 *$ \\
\hline & Não & 52 & 53,1 & 63 & 61,2 & & & \\
\hline \multirow{2}{*}{$\begin{array}{l}\text { Glicemia de jejum } \\
\text { (mg/dl) }\end{array}$} & Média & 105,4 & & 93,6 & & - & - & $0,02^{\# \#}$ \\
\hline & Desvio padrão & 36,2 & & 22,3 & & & & \\
\hline \multirow{2}{*}{$\begin{array}{l}\text { Insulina basal } \\
(\mathbf{m U I} / \mathbf{m l})\end{array}$} & Mediana & 9,3 & & 6,5 & & - & - & $<0,01^{s}$ \\
\hline & $\begin{array}{l}\text { Desvio } \\
\text { interquartílico }\end{array}$ & 12,2 & & 5,3 & & & & \\
\hline \multirow{2}{*}{$\begin{array}{l}\text { HOMA-IR } \\
\text { (Qualitativo) }\end{array}$} & $>3,8$ & 19 & 31,1 & 2 & 2,8 & 15,83 & 4,72 a 53,14 & $<0,01^{\#}$ \\
\hline & $<3,8$ & 42 & 68,9 & 70 & 97,2 & & & \\
\hline \multirow{2}{*}{$\begin{array}{l}\text { HOMA-IR } \\
\text { (Quantitativo) }\end{array}$} & Mediana & 2,34 & & 1,47 & & - & - & $<0,01^{s}$ \\
\hline & $\begin{array}{l}\text { Desvio } \\
\text { interquartílico }\end{array}$ & 3,55 & & 1,18 & & & & \\
\hline
\end{tabular}

TESTES: * Qui-quadrado, ** Qui-quadrado de tendência, ${ }^{*}$ Exato de Fisher, ${ }^{\# \#}$ t de Student, ${ }^{s}$ Mann-Whitney 
A maior associação com insulinemia do que com glicemia de jejum, como se constatou no presente estudo, foi também observada por Norris e col. ${ }^{20} \mathrm{e}$ reforça $\mathrm{o}$ argumento de que acrocórdons podem representar, com pseudoacantose nigricans, marcadores de RI antes do surgimento de doenças decorrentes da síndrome hipermetabólica, uma vez que apenas $15 \%$ dos pacientes com RI não apresentam manifestações cutâneas."

A insulina é um hormônio promotor de crescimento tecidual que estimula a captação de glicose pelos tecidos em uma intensidade variável entre diferentes indivíduos. Quando existe RI, as células respondem menos à ação desse hormônio. Em compensação, o pâncreas passa a produzir maiores quantidades de insulina. ${ }^{1,9,21} \mathrm{O}$ hiperinsulinismo promove aumento do IGF-1 e redução da IGFBP-3 (insulin-like growth factor-binding protein 3), a qual é um dos ligantes do receptor nuclear de retinóide $\mathrm{X}$ tipo $\alpha$ $(\mathrm{RXR} \alpha)$, responsável pela transcrição de genes antiproliferativos. Tanto o hiperinsulinismo como o aumento do IGF-1 induzem diretamente o crescimento epitelial e fibroblástico a partir da ativação de receptores, podendo justificar a prevalência de acrocórdons e pseudoacantose nigricans nesses grupos. ${ }^{1}$

Barghava e Mathur observaram associação entre múltiplos acrocórdons, pseudoacantose nigricans, queratoses seborreicas, obesidade e intolerância à glicose $\mathrm{e}$ sugeriram que isso representa uma síndrome decorrente da secreção de fatores de crescimento tecidual. ${ }^{22,23}$

A alta prevalência de acrocórdons entre acromegálicos reforça a importância de fatores de crescimento na gênese e no desenvolvimento dessas lesões, visto que tais pacientes expressam maiores níveis de insulina, hormônio do crescimento (GH) e IGF-1. ${ }^{24}$

Observou-se, igualmente, maior marcação de receptores de EGF no epitélio de acrocórdons entre portadores de síndrome do nevo displásico, aventando-se que essa expressão tecidual poderia ser induzi- da, ainda, por outros fatores de crescimento tecidual circulantes, além do IGF-1 e da insulina. Entretanto, níveis de fatores de crescimento, como o $\mathrm{GH}$, não foram investigados, até o momento, entre pacientes saudáveis portadores de acrocórdons, tampouco nos portadores de síndrome do nevo displásico. ${ }^{25}$

A coloração dos acrocórdons não foi associada a nenhuma característica clínica e é provável que reflita aspectos relacionados à pigmentação constitucional. ${ }^{1,9} \mathrm{Em}$ um estudo indiano ${ }^{22} \mathrm{e}$ em outro israelense, ${ }^{26}$ a maioria dos acrocórdons foi referida como hipercrômicos, o que não se observa em estudos ocidentais.

Assim como evidenciado neste estudo, outros autores associaram acrocórdons a fatores de risco cardiovasculares, como a dislipidemia e a obesidade, apontando para um risco maior de doença aterosclerótica. ${ }^{22,27}$ Outras alterações relacionadas à RI incluem a hiperuricemia, níveis elevados do inibidor do ativador do plasminogênio 1, aumento do IGF-1 e maior quantidade de partículas pequenas de LDL, fatores que também se associam à aterosclerose coronariana. ${ }^{12,28}$ Há diversos achados dermatológicos que se relacionam especialmente à aterosclerose. Nesse sentido, acrocórdons também deveriam ser investigados, o que poderia colaborar com a estratificação de risco da população. ${ }^{29}$

Outros estudos que avaliaram pacientes com acrocórdons identificaram alterações do metabolismo da glicose entre $28 \%$ e $81 \%$ dos pacientes, dependendo da população estudada, o que se correlaciona com os $41 \%$ encontrados na presente casuística. ${ }^{7,19,22,27}$

Alguns autores correlacionaram o número total de acrocórdons com níveis de glicemia de jejum e com IMC, porém, essas relações ainda permanecem controversas. ${ }^{1,79,20,26,27}$

A topografia das lesões não prediz alteração do metabolismo de carboidratos, como foi evidenciado neste estudo; no entanto, mulheres apresentaram mais lesões inframamárias do que homens, o que deve ocorrer pela proeminência das mamas. ${ }^{1,7,920,26,27}$

TABela 2: Modelo logístico multivariado ajustado pelas covariáveis selecionadas*

\begin{tabular}{llll}
\hline Variável & OR & IC 95\% & p \\
\hline HOMA-IR (Quantitativo) & 1,39 & 1,03 a 1,87 & 0,03 \\
IMC $\left(\mathrm{kg} / \mathrm{m}^{2}\right)$ & 1,13 & 1,03 a 1,24 & 0,01 \\
Diabetes mellitus & 3,00 & 0,83 a 10,94 & 0,10 \\
Diabetes mellitus familiar & 0,32 & 0,12 a 0,83 & 0,02 \\
Idade (anos) & 0,97 & 0,93 a 1,01 & 0,10 \\
Hipertrigliceridemia & 3,67 & 1,30 a 10,39 & 0,01 \\
fototipo I e II** & 1 & - & 0,07 a 1,31 \\
$\quad$ III e IV & 0,30 & 0,23 a 3,73 & 0,11 \\
V e VI & 0,92 & - & 0,18 \\
\hline
\end{tabular}

$*$ p do modelo completo $<0,01$. R2 Nagelkerke $=0,43 * *$ Variável de referência ordinal 
Em outro estudo, ${ }^{9}$ múltiplos acrocórdons predisseram RI com mais sensibilidade do que a presença de pseudoacantose nigricans. O desenho deste trabalho não permitiu essa comparação em relação à RI, pois a presença de mais de cinco acrocórdons foi o fator de seleção dos pacientes (variável dependente); contudo, acrocórdons se mostraram marcadores de RI independentemente da presença de pseudoacantose nigricans.

Pacientes adultos com acrocórdons devem ser alertados para o risco de desenvolver RI, hipertrigliceridemia, sobrepeso e, possivelmente, DM e as complicações cardiovasculares associadas, como infarto agudo do miocárdio, doença cerebrovascular, doença arterial periférica, disfunção erétil, declínio cognitivo, esteatose hepática e doença renovascular. Além disso, a RI tem sido associada ao desenvolvimento de neoplasias malignas como adenocarcinoma de cólon, mama, endométrio, rim, esôfago, próstata, o que pode se justificar pelos maiores níveis de fatores de crescimento tecidual desses pacientes. ${ }^{11,30}$ No entanto, ainda não há pesquisas comparando a presença de acrocórdons e tais desfechos específicos.

Uso de medicamentos, doenças crônicas, atividade física, dieta e estado nutricional são fatores que podem influenciar diretamente a estimativa pontual da RI pelo índice HOMA-IR e não podem ser controlados adequadamente em estudos transversais. Por outro lado, a dimensão da associação entre RI e acrocórdons observada neste trabalho e corroborada pela literatura, mantida mesmo após ajuste pelas variáveis de confusão, além da identificação entre os casos de outros marcadores de RI, como IMC, DM e obesidade abdominal, não apoia a ideia de que a associação tenha ocorrido ou tenha sido marcadamente influenciada por esse viés.

\section{REFERÊNCIAS}

1. Rasi A, Soltani-Arabshahi R, Shahbazi N. Skin tag as a cutaneous marker for impaired carbohydrate metabolism: a case-control study. Int J Dermatol. 2007;46:1155-9.

2. Pariser RJ. Benign neoplasms of the skin. Med Clin North Am. 1998;82:1285-307.

3. Banik R, Lubach D. Skin tags: localization and frequencies according to sex and age. Dermatologica. 1987;174:180-3.

4. Sociedade Brasileira de Dermatologia. Perfil nosológico das consultas dermatológicas no Brasil. An Bras Dermatol. 2006;81:549-58.

5. Dianzani C, Calvieri S, Pierangeli A, Imperi M, Bucci M, Degener AM. The detection of human
Outro elemento que merece destaque é que os valores de referência do HOMA-IR não são consensuais na literatura, variando de 2,7 a 8,2 , de acordo com características étnicas, IMC e idade. Os dados deste estudo foram testados para os diferentes pontos de corte e mostraram significância em todos os níveis. No laboratório da instituição, adota-se o limite de 3,8, empregado aqui. Por outro lado, a identificação da associação entre acrocórdons e níveis quantitativos de HOMA-IR ajustados para idade e IMC minimiza o viés advindo da adoção de pontos de corte específicos.

Há carência de estudos de base populacional que caracterizem os acrocórdons, seus aspectos clínicos e laboratoriais, a história natural, os elementos prognósticos, além de possíveis particularidades étnicas e culturais. Sua associação com RI, além de corroborar o conhecimento sobre a fisiopatogênese da doença, deve incentivar a realização de outras investigações com casuística mais expressiva, que permita a ponderação de outras variáveis, a fim de precisar a estimativa de risco e a estratificação de populações. Estudos prospectivos possibilitariam mensurar, ainda, índices de mortalidade geral e específica. Da mesma forma, é importante explorar o significado de acrocórdons em diferentes topografias corporais, número e cor, além do impacto das medidas de modificação (farmacológicas ou não) da RI na sua gênese.

\section{CONCLUSÃO}

Em pacientes ambulatoriais dermatológicos, a presença de múltiplos acrocórdons se associou à resistência insulínica, ao sobrepeso e à hipertrigliceridemia, independentemente dos demais fatores de risco conhecidos.

papillomavirus DNA in skin tags. $\mathrm{Br} \mathrm{J}$ Dermatol. 1998; 138:649-51.

6. Erdo an BS, Aktan S, Rota S, Ergin S, Evliyao D. Skin tags and atherosclerotic risk factors. J Dermatol. 2005;32:371-5.

7. Margolis J, Margolis LS. Skin tags - a frequent sign of diabetes mellitus. $N$ Engl J Med. 1976;294:1184.

8. Mathur SK, Bhargava $P$. Insulin resistance and skin tags. Dermatology. 1997; 195:184.

9. Sudy E, Urbina F, Maliqueo M, Sir T. Screening of glucose/insulin metabolic alterations in men with multiple skin tags on the neck. J Dtsch Dermatol Ges. 2008;6:852-5. 
10. Piette AM, Meduri B, Fritsch J, Fermanian J, Piette JC, Chapman A. Do skin tags constitute a marker for colonic polyps? A prospective study of 100 asymptomatic patients and metaanalysis of the literature. Gastroenterology. 1988;95:1127-9.

11. Carvalheira JBC, Saad MJA. Doenças Associadas à Resistência à Insulina / Hiperinsulinemia, não Incluídas na Síndrome Metabólica. Arq Bras Endocrinol Metab. 2006;50:360-7.

12. Bonora E, Formentini G, Calcaterra F, Lombardi S, Marini F, Zenari L et al. HOMA-estimated insulin resistance is an independent predictor of ardiovascular disease in type 2 diabetic subjects: prospective data from the Verona Diabetes Complications Study. Diabetes Care. 2002;25:1135-41.

13. Garcia Hidalgo L. Dermatological complications of obesity. Am J Clin Dermatol. 2002;3:497-506.

14. Kede MPV, Figueira AL, Porto JA. Manifestações cutâneas no diabetes mellitus. An Bras Dermatol. 1993;68:21-4.

15. Levine N. Brown patches, skin tags on axilla. Are this patient's velvety plaques related to his obesity and diabetes? Geriatrics. 1996;51:27.

16. Geloneze B, Vasquez AC, Stabe CF, Pareja JC, Rosado LE, Queiroz EC et al. HOMA1-IR and HOMA2-IR indexes in identifying insulin resistance and metabolic syndrome - Brazilian Metabolic Syndrome Study (BRAMS) Arq Bras Endocrinol Metab. 2009;53:281-7.

17. McAuley KA, Williams SM, Mann JI, Walker RJ, Lewis-Barned NJ, Temple LA et al. Diagnosing insulin resistance in the general population. Diabetes Care. 200;24:460-4.

18. SPSS.com [homepage]. Statistical Package for Social Science (SPSS). Release Version 17.0.1. Chicago (IL): SPSS Incorporation; 2008. [cited 2009 Jul 10]. Available from: http://www.spss.com.

19. Agarwal JK, Nigam PK. Acrochordon: a cutaneous sign of carbohydrate intolerance. Australas J Dermatol. 1987;28:132-3.

20. Norris PG, McFadden J, Gale E, Griffiths WA. Skin tags are more closely related to fasting insulin than fasting glucose levels. Acta Derm Venereol. 1988;68:367-8.

21. Hermanns-Lê T, Scheen A, Piérard GE. Acanthosis nigricans associated with insulin resistance: pathophysiology and management. Am J Clin Dermatol. 2004;5:199-203.
22. Bhargava P, Mathur D. Acrochordon, diabetes and associations. Indian J Dermatol Venereol Leprol. 1996;62:226-8.

23. Ellis DL, Kafka SP, Chow JC, Nanney LB, Inman WH, McCadden ME et al. Melanoma growth factors, acanthosis nigricans, the sign of Leser-Trelat and multiple acrochordons. N Engl J Med. 1987;317:1582-7.

24. Ben-Shlomo A, Melmed S. Skin manifestations in acromegaly. Clin Dermatol. 2006;24:256-9.

25. Ellis DL, Nanney LB, King LE Jr. Increased epidermal growth factor receptors in seborrheic keratoses and acrochordons of patients with the dysplastic nevus syndrome. J Am Acad Dermatol. 1990;23:1070-7.

26. Kahana M, Grossman E, Feinstein A, Ronnen M, Cohen M, Millet MS. Skin tags: a cutaneous marker for diabetes mellitus. Acta Derm Venereol. 1987;67:175-7.

27. Demir S, Demir Y. Acrochordon and impaired carbohydrate metabolism. Acta Diabetol. 2002;39:57-9.

28. Crook MA. Skin tags and the atherogenic lipid profile. J Clin Pathol. 2000;53:873-4.

29. Miot HA, Medeiros LM, Siqueira CRS, Cardoso LC, Gumieiro JH, Pandini Filho MA et al. Associação entre doença arterial coronariana $\mathrm{e}$ as pregas lobular diagonal e anterotragal em homens. An Bras Dermatol. 2006;81:29-33.

30. Hsing AW, Gao YT, Chua S JR, Deng J, Stanczyk FZ. Insulin resistance and prostate cancer risk. J Natl Cancer Inst. 2003;95:66-71.

Como citar este arquivo/How to cite this article: Tamega AA, Aranha AMP, Guiotoku MM, Miot LDB, Miot HA. Associação entre acrocórdons e resistência à insulina. An Bras Dermatol. 2010;85(1):25-31. 